\title{
La fabrication des composants en verre optique
}

\author{
Christian BEURTHE \\ Laboratoire Charles Fabry de l'Institut d'Optique \\ christian.beurthe@institutoptique.fr \\ Avec l'aimable collaboration de Raymond MERCIER
}

Cet article traite des différentes méthodes d'usinage et de polissage des verres optiques. Dans un premier temps, il décrit comment mettre en forme un verre optique : cette étape est incontournable et est toujours d'actualité dans tous les ateliers de fabrication. Ensuite, il explique comment polir et obtenir, par la méthode traditionnelle de polissage sur un support de poix, aussi bien la régularité que la rugosité des surfaces optiques.

Enfin, deux autres méthodes seront abordées : ce sont des méthodes de finition, permettant d'obtenir de hautes précisions au niveau de la régularité ou de l'asphérisation des surfaces.

\section{L'usinage du verre}

Le verre est un matériau non-cristallin présentant le phénomène de transition vitreuse : il est dur et cassant. II n'est pas usinable comme les matériaux ductiles (par exemple les métaux) en enlevant de la matière avec un outil coupant, mais uniquement par abrasion.

Qu'est-ce que l'abrasion ? C'est l'action qui consiste à réaliser des micro-cassures dans la matière, en faisant un apport d'abrasif libre sur un support en rotation. Il faudra diminuer l'amplitude des micro-cassures par des étapes successives d'usure avec des abrasifs de plus en plus fins, jusqu'à obtenir une surface polie.

L'usinage du verre comporte quatre étapes indissociables : le découpage, l'ébauchage, le doucissage et le polissage.

Le découpage (figure 1) est la mise en forme grossière de la pièce à fabriquer. Elle s'effectue soit avec une roulette de carbure, dans le cas de découpe de planimètre (float glass) comme tous les vitriers le font, soit avec une scie munie d'un disque diamanté, en milieu aqueux pour éviter les cassures du verre par échauffement lors de la coupe.

L'ébauchage est la mise en forme de l'objet à fabriquer : à ce stade, les précisions obtenues sont de l'ordre du centième de millimètre sur la cotation, de quelques microns sur la régularité des surfaces et d'une vingtaine de microns sur la rugosité des surfaces. L'ébauchage est réalisé manuellement sur un outil tournant en fonte, préalablement rectifié, soit plan, soit du rayon désiré. Cette action s'effectue en insérant de l'abrasif en grain libre entre la pièce à usiner et l'outil. La grosseur des abrasifs d'ébauchage varie de cent cinquante microns à quarante microns. Pour arriver à une surface finie à

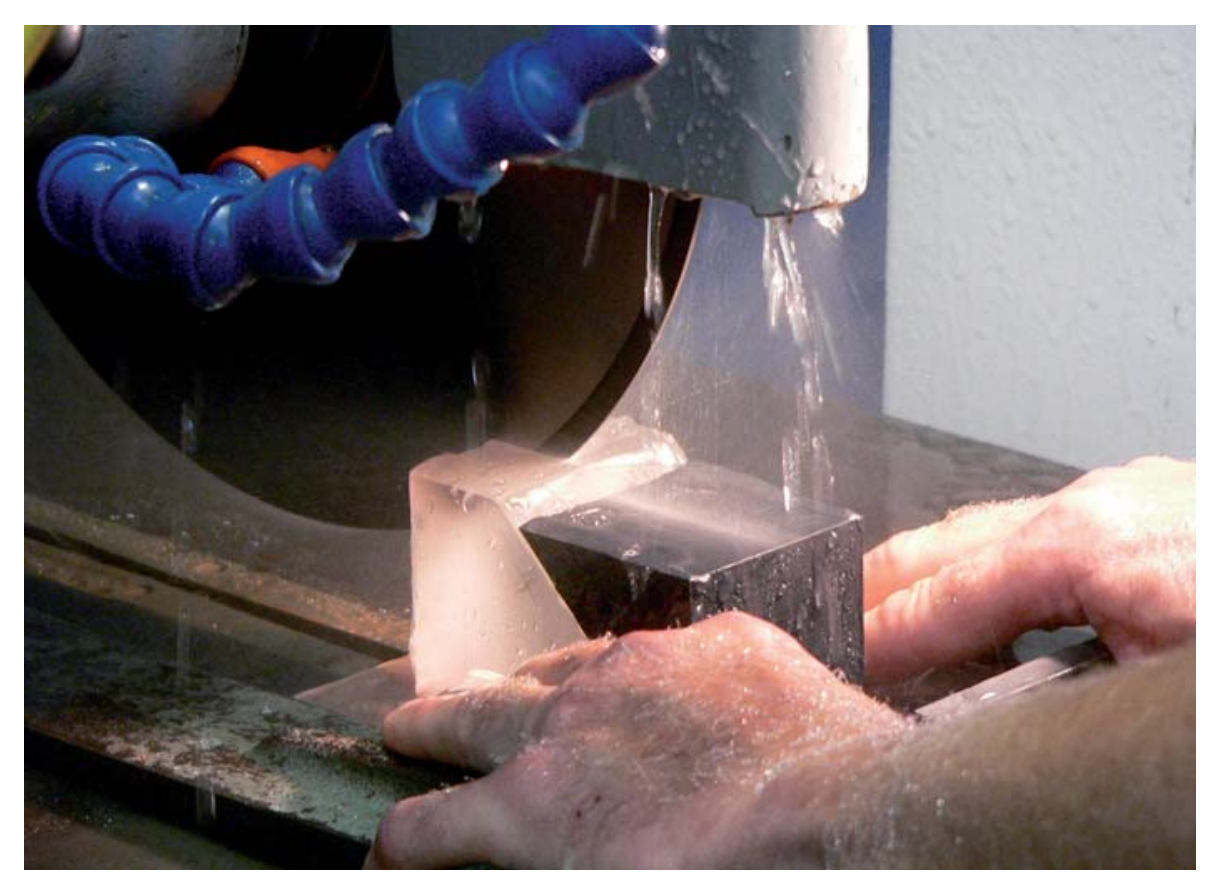

Figure 1. Le découpage du verre, ici par disque diamanté, permet de donner au composant sa forme grossière. ce stade, il faut toujours utiliser les abrasifs par grosseur décroissante, et récupérer la rugosité de l'abrasif le plus gros, par l'abrasif de granulométrie inférieure.

Le doucissage est toujours réalisé sur un outil tournant, mais cette fois-ci dans une matière plus tendre. Le choix se porte traditionnellement sur un outil en laiton car les abrasifs à cette étape sont beaucoup plus fins et risquent de rayer la surface à usiner. Au stade précédent

Figure l. Le découpage du verre, icipar disque diamanté, permet de donner au composantsa formegrossière. 


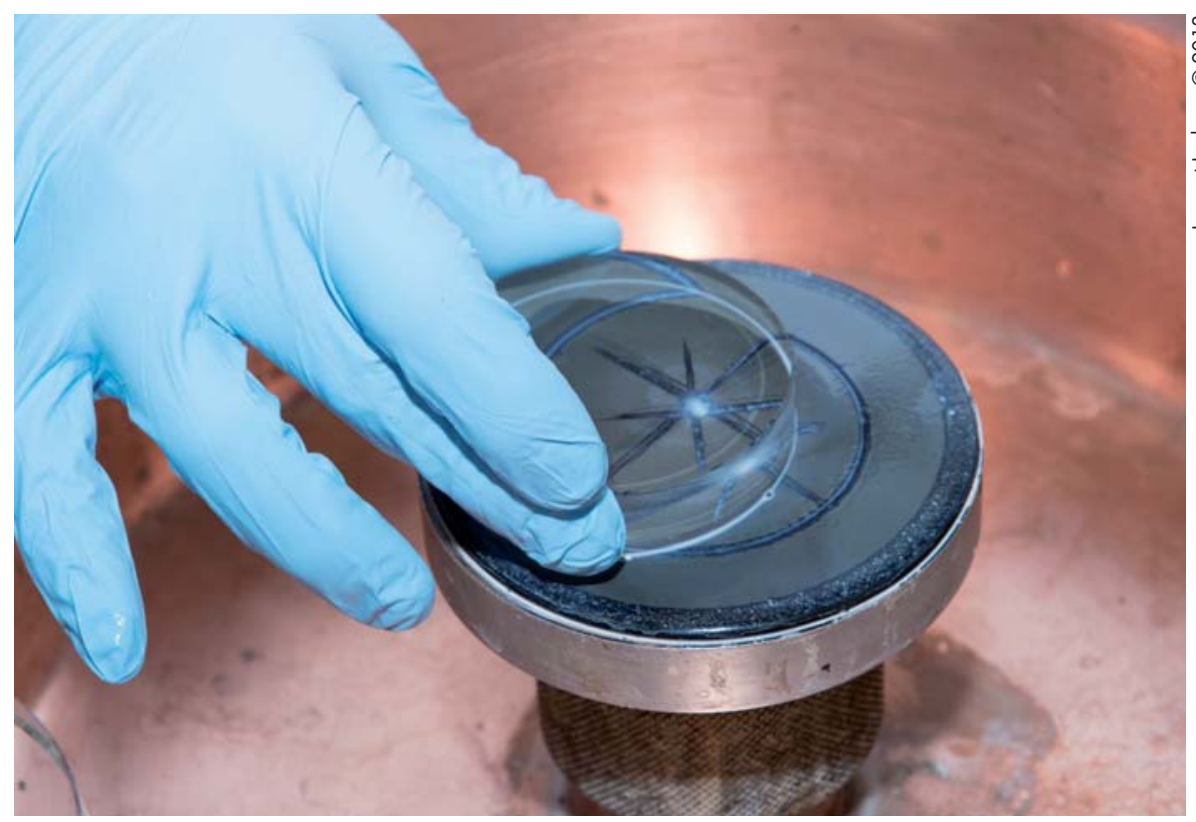

Figure 2. Le polissage avec un support en poix est exclusivement employé en optique de précision.

(l'ébauchage), l'abrasif final est de quarante microns; au doucissage les grosseurs de grain employées, toujours par ordre décroissant, vont de 15 à 3 microns.
Quand la surface du composant sera terminée à l'abrasif d'une grosseur de trois microns, elle ne sera toujours pas polie, mais deviendra translucide. Le doucissage permet d'amener la surface à une rugosité si fine qu'elle permet ensuite d'effectuer le polissage. Parallèlement, l'emploi d'abrasifs fins permet d'affiner les précisions sur le parallélisme, la cotation...

Le polissage est de deux types. Le polissage dit « rapide » s'exécute sur un support tournant en aluminium rectifié sur lequel est collé un textile de polissage autocollant, comme il en existe pour la métallographie. Pour que le polissage puisse se faire, il faut un abrasif très fin et de dureté supérieure au verre : I'oxyde de cérium est utilisé de façon courante. $\mathrm{Ce}$ type de polissage est employé quand les qualités de surface demandent quelques nanomètres de rugosité et une centaine de nanomètres de régularité. Ce polissage « rapide » est très largement employé pour la lunetterie.

La deuxième qualité de polissage, de loin la plus difficile à mettre en œuvre, est le polissage avec un support en poix. $\mathrm{Ce}$ polissage est exclusivement employé en optique de précision (figure 2).

\section{HORIBA}

Scientific

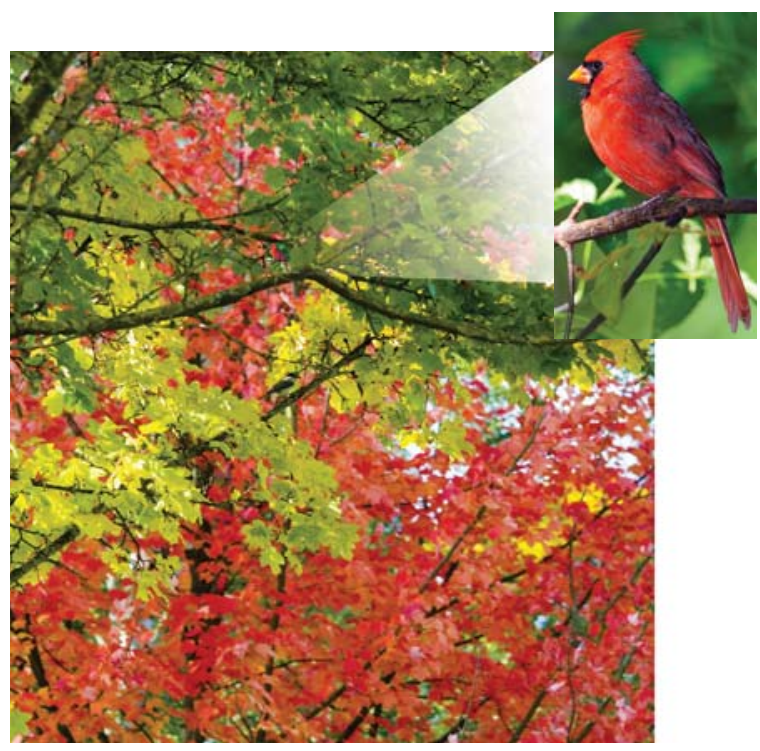

\section{Avoir une vue d'ensemble} n'est pas toujours suffisant

Si vous souhaitez voir les détails, vous avez besoin du spectromètre d'ellipsométrie le plus avancé,

\section{|'UVISEL 2.}

- Plusieurs tailles de spot à partir de $35 \mu \mathrm{m}$

- Visualisation efficace de la zone de mesure

- Plateforme de métrologie automatisée

- Large gamme spectrale de 190 à 2100 nm haute résolution

- Suite logicielle Delta Psi 2

- Technologie performante et rapide à modulation de phase

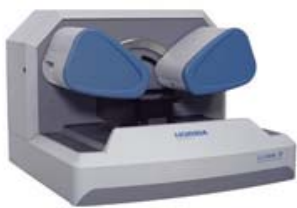




\section{Le polissage avec support de poix}

Qu'est-ce que la poix ? C'est une résine issue de certains résineux qui présente l'avantage de pouvoir obtenir des surfaces extrêmement fines en rugosité. Néanmoins, comme elle est thermoplastique, elle fluctue continuellement dans sa forme. Elle oblige donc l'opérateur à rectifier continuellement la forme du support par un mouvement adéquat. Ce polissage demande une grande expérience et une grande dextérité, l'opérateur doit « sentir » l'adhérence de la surface sur la poix. Pour mieux contrôler cette étape d'usinage, une technique consiste à fabriquer de façon systématique une surface complémentaire au support de polissage. Si la surface ne prend qu'en un point, elle « roule ", et cela alertera l'operateur sur le fait que son polissoir est plus convexe que la surface de son composant. À l'inverse, si lors du polissage son composant « pince», cela l'alertera sur le fait que son polissoir a une forme plus concave que son composant, ce qui impliquera que la surface de son composant aura tendance à passer dans le convexe. On comprend donc bien que ce polissage demande beaucoup de ressenti manuel. La régularité des surfaces n'a qu'un seul obstacle dans sa progression, c'est l'opérateur lui-même. Avec un opérateur de métier, il est possible d'obtenir une régularité de surface de quelques nanomètres et une rugosité d'un ou deux dixièmes de nanomètre. Pour contrôler ces surfaces, la technique utilisée est celle des franges d'interférence par rapport à une référence polie. La question qui se pose alors est la suivante : comment avoir une référence d'une telle précision, permettant de contrôler les surfaces ?

\section{La fabrication}

\section{des références de contrôle}

L'opticien de précision fabrique luimême ses références de contrôle. Pour fabriquer des références planes dites " étalon ", il utilise un moyen infaillible. Quand deux surfaces sont contrôlées sans observer d'écart via les franges d'interférence, on peut simplement dire qu'elles

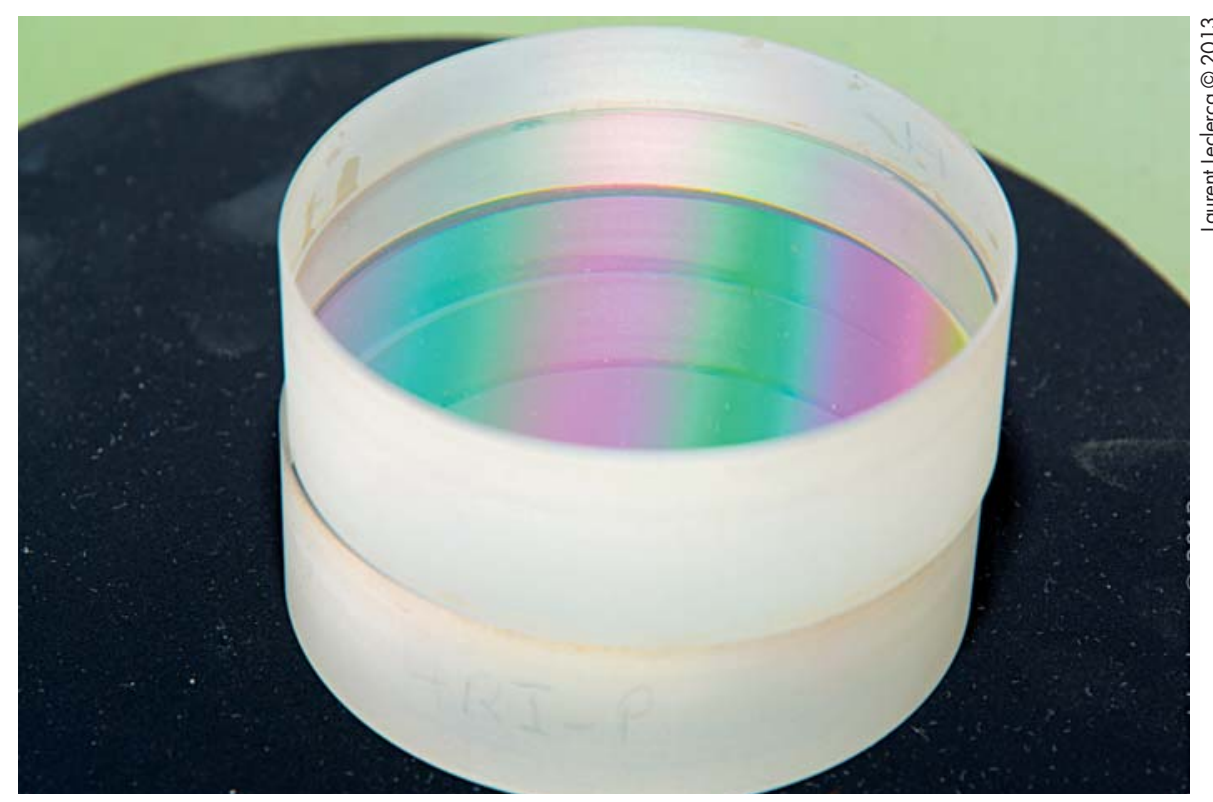

Figure 3. L'observation des franges d'interférence permet de contrôler une surface par comparaison avec un étalon de référence.

sont complémentaires, les deux planes ou I'une concave, l'autre convexe, de même rayon : avec deux surfaces, il est donc impossible de vérifier la planéité. Par contre, si une troisième surface est fabriquée et que ces trois surfaces sont contrôlées deux à deux et si elles sont complémentaires, on peut alors affirmer que ces surfaces sont réellement planes.

Dans le cas de surfaces de rayon bien défini, la fabrication des références de contrôle fait intervenir deux calibres, un concave et un convexe. Le contrôle précis du rayon est effectué sur banc optique, appareillage qui mesure optiquement par l'intermédiaire d'un déplacement sur une règle graduée le rayon de courbure d'une surface concave. Cette mesure effectuée, l'opérateur pourra fabriquer la surface complémentaire, donc convexe à cette surface. La régularité des deux surfaces sera contrôlée lors du polissage en observant les franges d'interférence (figure 3).

\section{Le polissage \\ par méthodes industrielles}

Le polissage manuel requiert une très bonne habileté et beaucoup d'heures passées pour obtenir une qualité de surface inégalée, mais uniquement planes ou sphériques. C'est pour cela que certains industriels se sont tournés vers des méthodes de retouches finales des surfaces beaucoup plus industrielles.

\section{La magnétorhéologie}

Le composant est usiné et poli sur un support de poix par les méthodes traditionnelles, sans rechercher une qualité de régularité supérieure. La finition, qui, bien sûr, rime avec précision, est de loin l'étape qui demande le plus de temps. De plus en plus d'industriels, faisant de grandes séries de composants précis, se tournent vers l'usinage par magnétorhéologie.

Dans cette méthode dite aussi méthode MRF (figure 4), le composant est mis en rotation à distance fixe d'une roue métallique, elle aussi en rotation. Un électroaimant placé sous la surface de cette roue génère un champ magnétique. Un fluide de polissage chargé de particules métalliques est injecté sur cette roue, est mis en forme par le champ magnétique et sert d'outil de polissage. L'usure au polissage ne dépend que du temps passé en contact avec le fluide de polissage et est gérée numériquement.

\section{Usinage par faisceau d'ions}

Cette méthode est basée sur la capacité d'un faisceau d'ions à enlever de la matière sans dégrader la rugosité initiale. Développée par Kodak pour retoucher les 


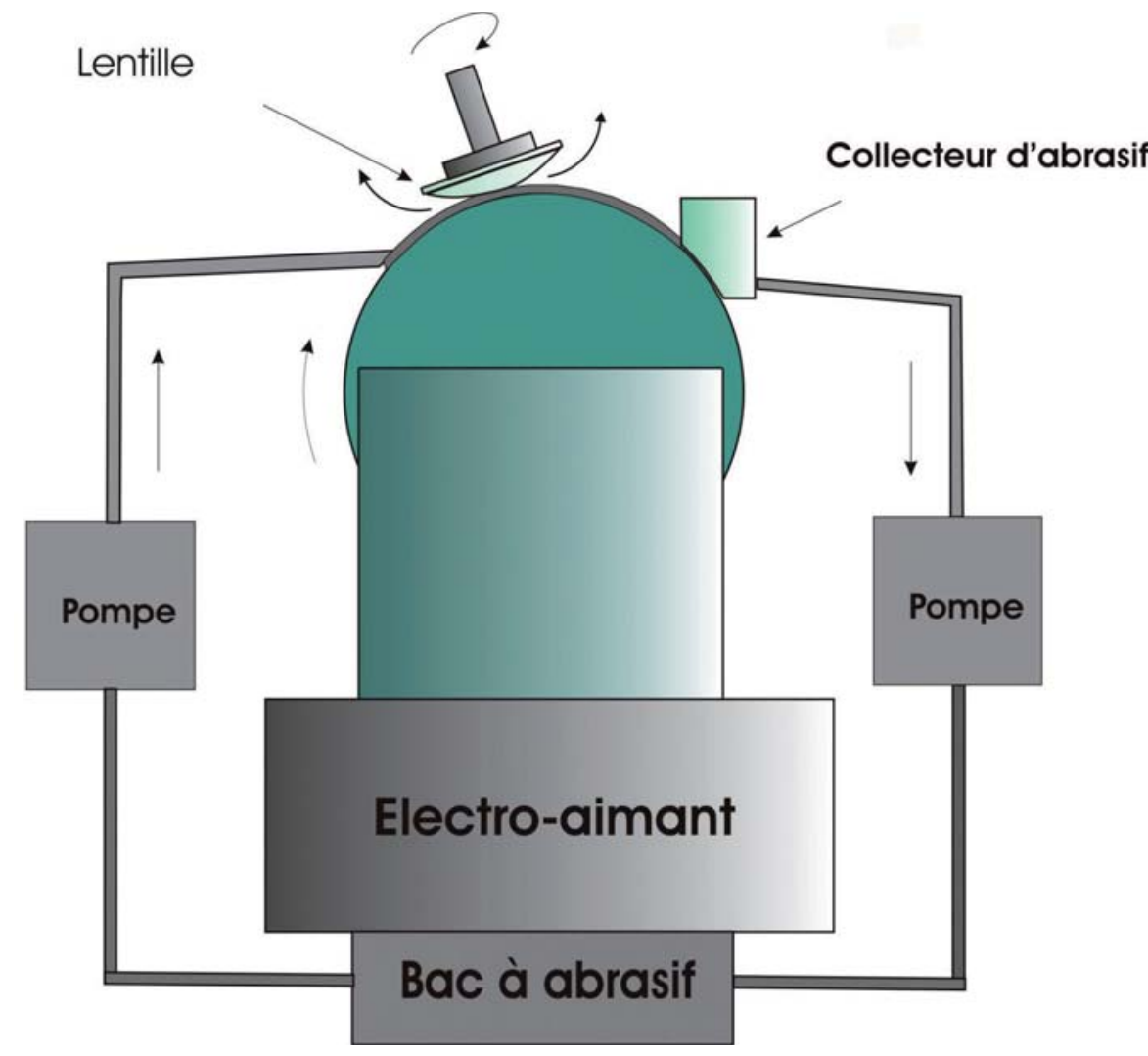

Figure 4. Schéma de principe de la magnétorhéologie, dite méthode MRF, permettant le polissage industriel.

miroirs allégés, sa version la plus répandue utilise un « petit faisceau », petit par rapport au substrat à retoucher. Comme pour la MRF, l'enlèvement de matière est déterminé par le temps de passage du faisceau sur la zone considérée. Le faisceau d'ions a l'avantage de présenter une excellente reproductibilité du taux d'enlèvement de la matière, ce qui assure une convergence rapide du procédé. Par contre, il a l'inconvénient d'obliger à travailler sous vide. Une autre version utilise un faisceau large, couvrant la totalité du substrat. Un masque découpé avec l'ouverture adéquate est placé entre la source d'ions et le substrat qui est en rotation uniforme, ce qui permet de moduler radialement le faisceau et ainsi de transformer une surface plane ou sphérique, polie classiquement sur poix donc avec une rugosité très faible (typiquement en dessous de 0,2 nm RMS), en surface asphérique avec la même rugosité. Contrairement aux méthodes précédentes, elle n'est pas adaptée pour retoucher les surfaces, mais elle permet de réaliser des asphérisations de forte amplitude, typiquement de la centaine de nanomètres à la dizaine de microns. Cette technique est particulièrement utile dans le domaine des courtes longueurs d'onde (extrême ultraviolet, de quelques nanomètres à quelques dizaines de nanomètres), pour des applications spatiales (physique solaire), ou d'imagerie sur synchrotron.

\section{Mieux connaître les technologies photoniques}

Dans chaque numéro, nous publions sous le titre «Comprendre » un point sur une technologie ou un phénomène optique. Les sujets choisis pour 2014 couvrent un large spectre, puisque, dans nos prochains numéros, nous aborderons successivement :

- Le couplage cohérent de lasers $\bullet$ L'ordinateur quantique $\bullet$ La photonique intégrée sur silicium • Les sondes locales $\bullet$ L'holographie digitale

Vous souhaitez intervenir sur l'un ou l'autre de ces sujets ou proposer à notre comité de rédaction un thème pour les numéros suivants?

N'hésitez pas à contacter notre rédaction : photoniques@edpsciences.org

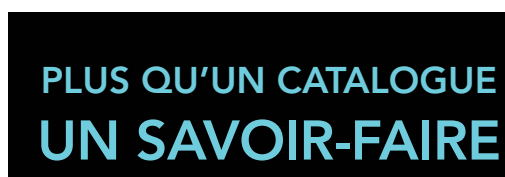

- Plus de 26500 PRODUITS EN STOCK

- Possibilités de fabrication SUR MESURE

- REMISES sur grandes quantités

- SUPPORT TECHNIQUE en français
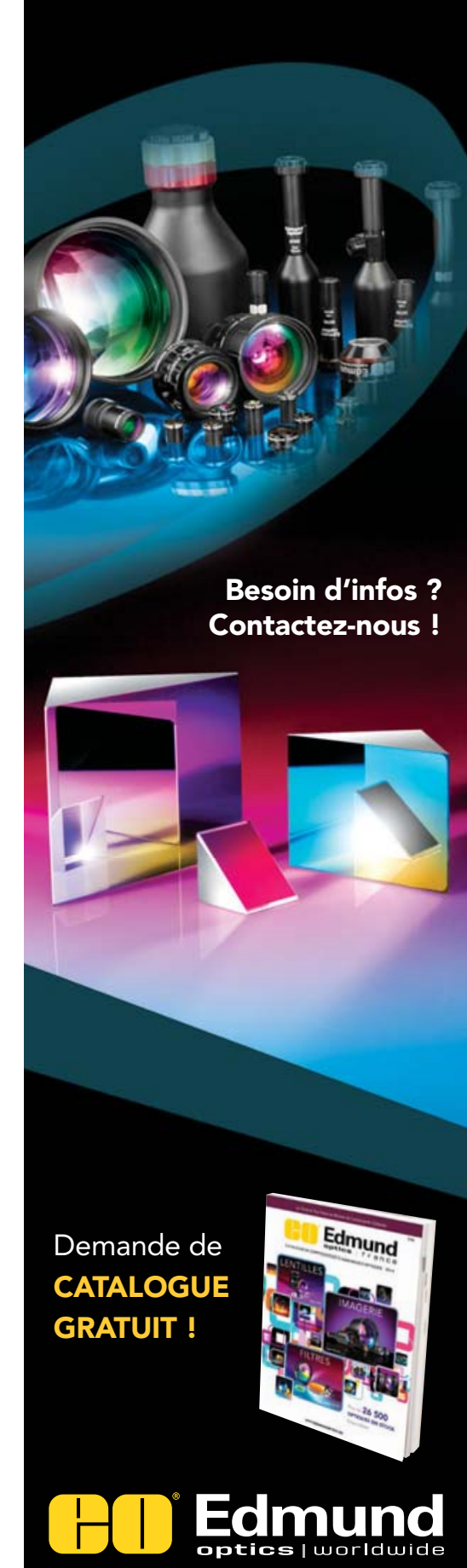

www.edmundoptics.fr

+33 (0)8 20207555 | sales@edmundoptics.fr 